\title{
A compact device for simultaneous dielectric spectroscopy and microgravimetric analysis under controlled humidity
}

\author{
Alessia Gennaro' ${ }^{1}$ Sebastian Rosa², Peter Cornelis ${ }^{1}$, Helge Pfeiffer ${ }^{3}$, E. Anibal \\ Disalvo ${ }^{2}$, Patrick Wagner ${ }^{1}$, Michael Wübbenhorst ${ }^{1}$
}

\author{
${ }^{I}$ KU Leuven, Department of Physics and Astronomy, Laboratory for Soft-Matter Physics and Biophysics \\ (ZMB), Celestijnenlaan 200 D, 3001 Leuven, Belgium \\ ${ }^{2}$ Laboratorio de Biointerfases y Sistemas Biomimeticos, Centro de Investigacion y Transferencia de Santiago \\ del Estero, Universidad Nacional de Santiago del Estero-Consejo Nacional de Investigaciones Científicas y \\ Técnicas, Santiago del Estero, Argentina \\ ${ }^{3}$ KU Leuven, Department of Materials Engineering (MTM), Kasteelpark Arenberg 44, 3001 Leuven, Belgium
}

(Presented XXXXX; received XXXXX; accepted XXXXX; published online XXXXX)

(Dates appearing here are provided by the Editorial Office)

\begin{abstract}
Water plays a key role in the functioning of natural and synthetic molecular systems. Despite several hydration studies, different techniques are employed individually for monitoring different physical features such as kinetics, dynamics and absorption. This study describes a compact hydration cell, which enables simultaneous dielectric relaxation spectroscopy (DRS) and mass loss/uptake measurements in thin organic layers under controlled humidity conditions and in a wide temperature range. This approach enabled us to correlate the physical quantities obtained during the same experiment by complementary techniques. To demonstrate the performance of this device, a $200 \mathrm{~nm}$ thick Poly(methyl methacrylate) (PMMA) layer was measured at various relative humidity levels $(0-75 \%)$, temperatures $\left(25-75^{\circ} \mathrm{C}\right)$ and frequencies (DRS: $\left.0.1 \mathrm{~Hz}-1 \mathrm{MHz}\right)$ to study how hydration and dehydration processes affect its molecular dynamics. The results show the capability of this setup to study the structural changes upon variation of the water content.
\end{abstract}

\section{INTRODUCTION}

Hydration of natural or synthetic macromolecules is responsible for a variety of phenomena, governing both the structure and the stability of such systems. Studying the effect of hydration enhances the understanding of many complex biological mechanisms such as protein folding [1-3], phospholipid hydration, as well as the dynamics and conformation of polymers [4-5]. Especially, the hydration of biomolecules is extensively studied in relation to the dynamics, structure and function [6-8]. These systems exhibit relaxation features typical of polymer systems and glass-forming liquids.

Furthermore, hydration plays a complex functional role in living cells. Since the hydration water determines the membrane stability and regulates its interaction with other molecules such as ions and proteins, several experiments and simulations have been performed. Numerical simulations have been extensively applied for studying a broad range of dynamical properties, including the rotational, translational, and hydrogen-bond dynamics, in the hydration shell that consists of the first water layer surrounding the biomolecules and interacting with them [9]. Moreover, several methods provided information about the organization of the hydration states that is not only restricted to the membrane plane but to a region extending into the cytoplasm, in which polar head groups play a relevant role [10].

Previous studies have characterized the dynamical properties of water confined in phospholipid membranes and show that water dynamics slows down dramatically as the hydration decreases

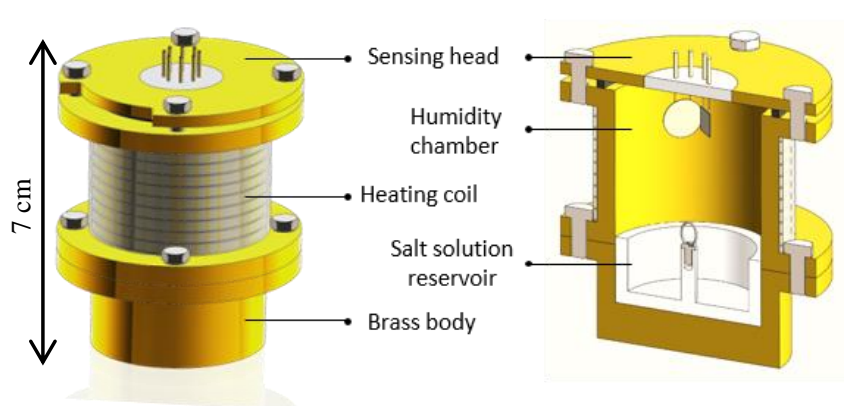

FIG. 1. Schematic illustration and cross-section of the custom-made hydration cell.

[11]. Another interesting phenomenon that has recently been studied is dehydration in a mixture of phospholipids and zwitterionic surfactants due to the formation of an inner salt layer between the dipoles of phospholipid and surfactant headgroups that reduces potential water binding sites [12].

Previous hydration studies aiming for example at a further refinement of hydration forces theory, largely relied on combining individual measurements of relevant physical quantities under controlled hydration conditions [13]. This procedure is both timeconsuming and challenging with respect to the accuracy of hydration control.

In this study, we propose an alternative approach that allows to simultaneously study the dielectric properties, including 
relaxation phenomena, and the water uptake/loss of a thin organic (polymer) film. In a compact, single setup providing quasiequilibrium humidity conditions, dielectric and gravimetric measurements at different temperatures are combined. To achieve this approach, we designed a compact hydration cell based on the following concepts:

a. Sealed, small volume hydration cell that contains all sensors, for the dielectric and microgravimetric measurements, together with a reservoir filled with a saturated aqueous salt solution. By choosing a specific salt, a desired relative humidity level (RH) can be maintained over a wide temperature range, which is, for most inorganic salts, almost temperature-independent.

b. Thermal insulation of the entire cell along with slow temperature changes allows quasi-equilibrium conditions regarding moisture uptake or desorption by all samples and sensors during heating or cooling protocols.

c. Flexible cell design regarding the number of samples and physical parameters being measured. The design, which we discuss here allows measuring three physical phenomena, being kinetics, adsorption and dynamics.

To demonstrate the capabilities of the hydration cell, we have studied the dielectric properties of Poly(methyl methacrylate) (PMMA) as a well-documented reference material [14-15]. Among the variety of polymers that have been examined as a function of water content by dielectric relaxation spectroscopy (DRS), e.g. Poly(2-methoxyethyl acrylate) PMEA [16, 17] and poly(ethylene glycol) PEG [18], PMMA was chosen for its capability to absorb water and its significant sensitivity to humidity. Despite its hydrophobic nature, it can absorb 2\% w/w (weight percent) of water. Moreover, it is low-cost and it shows long-term stability and resilience to aqueous salt solutions [19-21]. It has been widely used as an active polymer, for example in optical humidity sensors [22-24], in chemical sensor for detecting analytes in both liquid and gaseous media, in molecular separation and in drug delivery as a microparticles matrix [25].

The compact device presented in this work enables real-time to follow water absorption and desorption in function of time and temperature. Furthermore, it allows monitoring changes in the sample thickness.

DRS measurements were employed to study the molecular dynamics, the physical network morphology changes and the interfacial effects due to the loss and uptake of water molecules [26]. In addition, the absorption and with it the absolute layer thickness is monitored with monolayer sensitivity via a quartz crystal microbalance (QCM) that consist of a thin quartz crystal plate with metal electrodes deposited on both the front and back sides. The QCM technique is versatile. It has been widely employed in biosensors for biodetection [27,28], for the study of interactions between molecules and lipids [29] and for monitoring phase transition in biomimetic membranes [30,31]. Among other applications it is also used in soft matter characterization for the determination of materials sensing properties [32].

\section{EXPERIMENT}

\section{A. Experimental setup}

The main components of the hydration cell are displayed in Figure 1. The device consists of three major parts: two metal blocks in brass and a sensing head with pins to connect the system to the analyzers. These parts combine to form a cylindrical

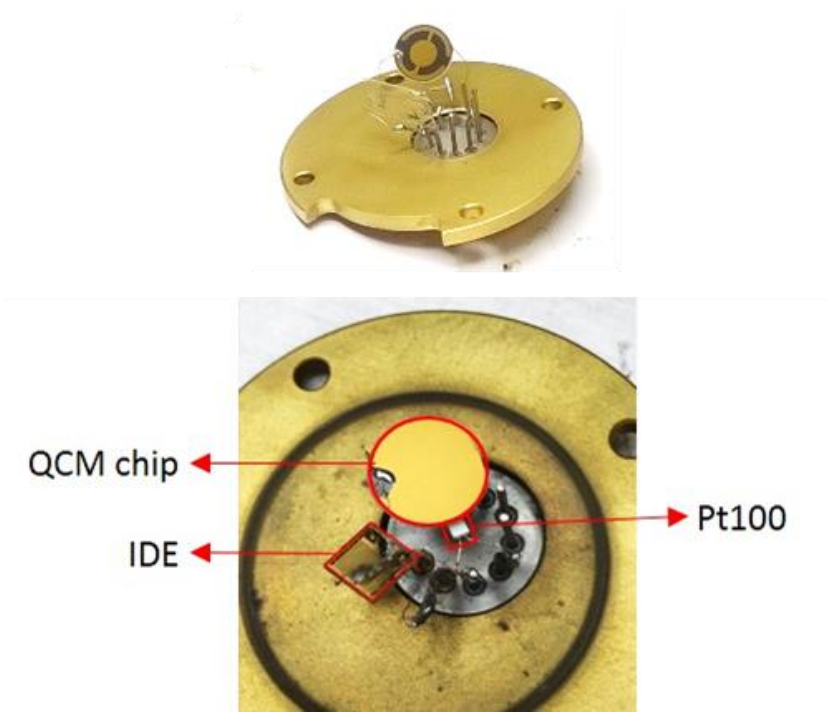

FIG 2. Photograph of the sensing head that includes a QCM chip, an IDE (interdigitated comb electrode) and a Pt100 resistor as temperature sensor.

humidity chamber, which is $7 \mathrm{~cm}$ deep with a $4 \mathrm{~cm}$ inner diameter. Moreover, in the bottom block a small bucket in polyether ether ketone (PEEK) is placed, which is filled with a saturated salt solution to control the relative humidity inside the chamber. It is a benefit that the bottom block can be mounted or dismounted separately to quickly change the salt solution without moving the sensing head. To control the inside temperature, a Thermocoax wire is coiled around the metal middle block and fixed with cement. The temperature range is between $21^{\circ} \mathrm{C}$ and $200^{\circ} \mathrm{C}$ at the current stage of development. All sensors in the system (QCM, interdigitated comb electrode IDE and Pt100) are mounted onto the brass cover plate as shown in Figure 2.

A conventional QCM sensor measures mass changes per unit area by measuring the change in frequency of the quartz crystal oscillator. The frequency increases or decreases respectively by the removal or addition of a small amount of mass from/onto the surface of the quartz sensing crystal. The Sauerbrey equation allows to convert the change of the resonance frequency in the mass change per unit area [33]:

$\Delta f=-\frac{2 f_{0}^{2}}{A \sqrt{\mu_{q} \rho_{q}}} \cdot \Delta m$

where $f_{0}$ is the resonant frequency of the fundamental mode of the QCM crystal, A is the area of the gold disk coated onto the crystal, $\mu_{\mathrm{q}}$ is the density of the crystal $\left(2.648 \mathrm{~g} \mathrm{~cm}^{-3}\right), \rho_{\mathrm{q}}$ is the shear modulus of the quartz $\left(1.67 \times 10^{5} \mathrm{~g} \mathrm{~cm}^{-1} \mathrm{~s}^{-2}\right)$ and $\Delta \mathrm{m}$ the change of the mass on the surface. For a given temperature and in the limit of a small sample mass, the frequency shift is directly proportional to the adsorbed mass on the QCM chip according to Eq.1.

In our setup the QCM chip is connected to a thickness counter (Tectra GmbH, Frankfurt, Germany). The raw data from the QCM chip are based on a constant water mass density of $1 \mathrm{~g} / \mathrm{cm}^{3}$ from which we obtained the apparent thickness variation due to water uptake and desorption. The mass change was calculated afterwards by multiplying the measured thickness by the water density. However, this density is temperature dependent, therefore the mass change has to be corrected using the water density values as tabulated by the NIST (National Institute of Standards and Technology) [34]. Once the mass variation has been determined, 
a)

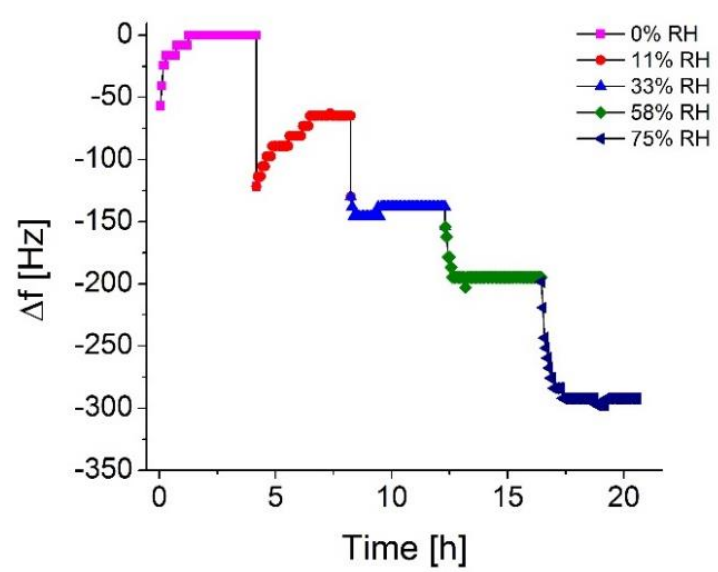

b)

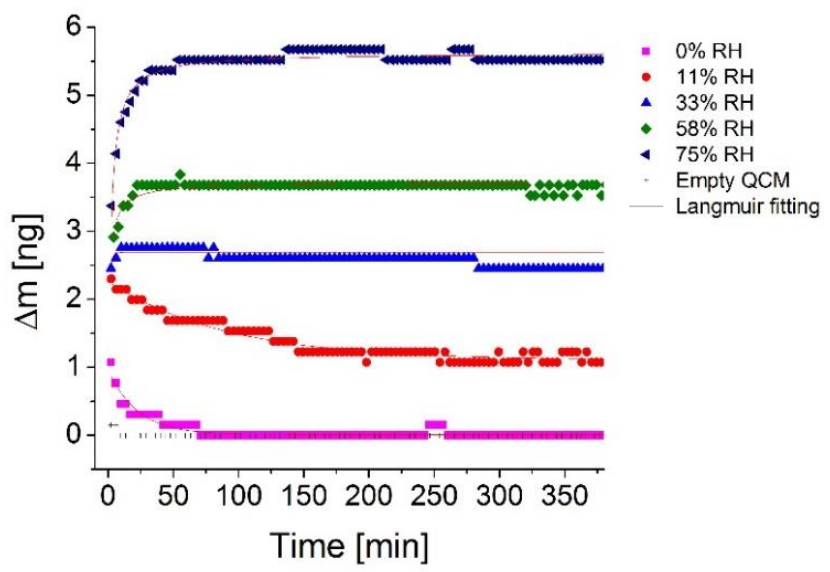

FIG. 3. (a) Frequency response calculated from the acquired apparent thickness of a PMMA coated QCM chip at constant relative humidity conditions between $0 \%$ and $75 \%$ RH using the Sauerbray model. (b) Water uptake kinetics at $25^{\circ} \mathrm{C}$ of a PMMA coated QCM chip, including fits based on the kinetic Langmuir adsorption isotherm model.

the frequency shift can be extracted by using Eq. 1. Hence it is possible to measure the film swelling and shrinking according to its moisture content.

Simultaneous dielectric spectra are acquired using $\mu \mathrm{m}$-spaced interdigitated comb electrodes in combination with a high resolution impedance analyzer (Alpha analyzer, Novocontrol Technologies). In the present work, IDEs have been designed using Clewin software and subsequently fabricated using optical lithography. Laser Writer LW405 (MICROTECH S.r.l., Palermo, Italy) was used for patterning planar structures in situ directly on the wafer. First, the glass substrate was coated with $100 \mathrm{~nm} \mathrm{Al}$ layer by sputter coating and then with the photoresist by spin coating. After development, the photoresist was removed by etching to make the exposed region transparent. The obtained IDEs have 100 pairs of comb-like shape electrodes $5 \mu \mathrm{m}$-spaced with a width of $5 \mu \mathrm{m}$ and a length of $3600 \mu \mathrm{m}$.

After soldering the sample to the pins, the sensing head is placed on top of the device to seal the humidity chamber. For this purpose, four screws are used to get a good seal by tightening an O-ring between the sensing head and the main body of the device. Furthermore, thermal convection effects caused by ambient air turbulence are minimized by putting the whole setup inside an insulating box.

Such IDE configurations have various advantages over the classical parallel-plate configuration [35,36]. As the electric field is planar, the total capacitance of the IDE can be easily decomposed into a background part related to the empty IDE having an intrinsic capacitance $\mathrm{C}_{0}$ and a variable component that depends on the film thickness. Conductive epoxy resin is used to glue silver wires onto the connect pads of the IDE.

\section{B. Sample preparation}

As a proof-of-concept, syndiotactic PMMA was used as the sensing layer. PMMA layers were synthesized by dissolving 0.1 $\mathrm{mg}$ of s-PMMA in $2 \mathrm{~g}$ of Chloroform. All reagents were used as received from Sigma-Aldrich N.V. (Diegem, Belgium) and had a purity of minimally $99.9 \%$. This mixture was stirred at $65{ }^{\circ} \mathrm{C}$ for 60 min to completely dissolve the polymer. In a next step, the solution was spin-coated $(60 \mathrm{~s}$ at $1000 \mathrm{rpm})$ onto a $1 \mathrm{~cm}^{2}$ aluminum-coated glass substrate and onto the QCM chip. This resulted in PMMA layers with an average thickness of $200 \mathrm{~nm}$ as measured using atomic force microscopy. Subsequently the samples were placed in the hydration cell and exposed to $\mathrm{P}_{2} \mathrm{O}_{5}(0 \%$ $\mathrm{RH})$ until equilibrium.

Four different saturated salt solutions were used to control the $\mathrm{RH}: \mathrm{LiCl}(13 \% \mathrm{RH}), \mathrm{MgCl}_{2}(33 \% \mathrm{RH}), \mathrm{NaBr}(58.2 \% \mathrm{RH})$ and $\mathrm{NaCl}(75 \% \mathrm{RH})$ [37]. Before each measurement the samples were equilibrated in $\mathrm{P}_{2} \mathrm{O}_{5}$ thus the dried state was the reference condition.

\section{QCM gravimetric measurements}

The QCM technique was used to analyse the absorptiondesorption kinetics of water on the PMMA film. The corresponding variation in the frequency shift reflects the mass uptake or loss. A gold quartz crystal (Inficon No. 008-010-G10) with a fundamental frequency of $6 \mathrm{MHz}$ in air was used as a mass sensor. When operating in its fundamental mode, the theoretical sensitivity factor is $16.02 \mathrm{ng} \mathrm{Hz}^{-1}\left(12.05 \mathrm{ng} \mathrm{Hz}^{-1} \mathrm{~cm}^{-2}\right)$ [38].

Isothermal measurements at $25{ }^{\circ} \mathrm{C}$ were performed for studying the water uptake/desorption kinetics after a step-like change in RH. Moreover, the effect of the temperature on the PMMA kinetics was monitored by implementing temperature ramps.

\section{Dielectric measurements}

Dielectric relaxation spectra in the frequency range from 0.1 to $10^{6} \mathrm{~Hz}$ were obtained by performing a heating and cooling cycle between $25^{\circ} \mathrm{C}$ and $75^{\circ} \mathrm{C}$ at a rate of $1 \mathrm{~K} / \mathrm{min}$. Generally, the complex dielectric permittivity, $\varepsilon^{*}$, contains information about the electronic, atomic and orientational polarizability of a dielectric material. While the fast, electronic contribution represents the permittivity at optical frequencies via the Maxwell relation $\varepsilon \approx \mathrm{n}^{2}$ ( $\mathrm{n}$ is the refractive index), the orientational polarization gives access to molecular relaxation processes, which occur on time- 
a)

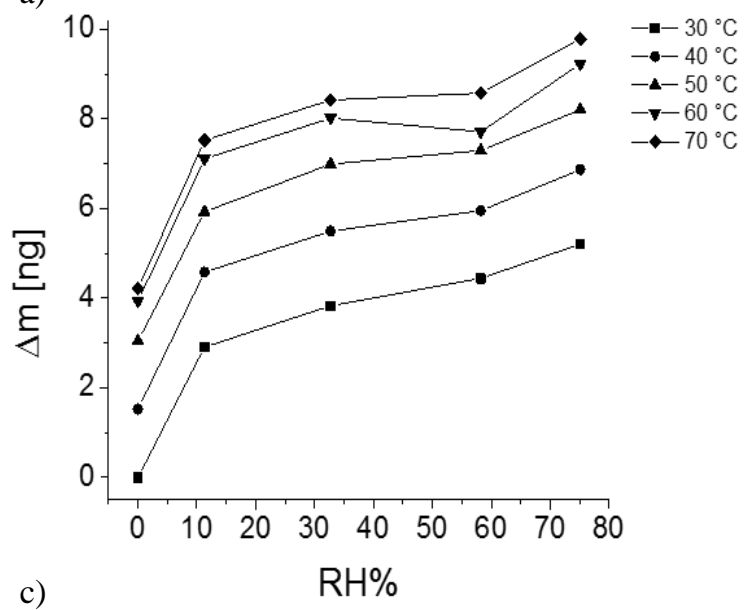

c)

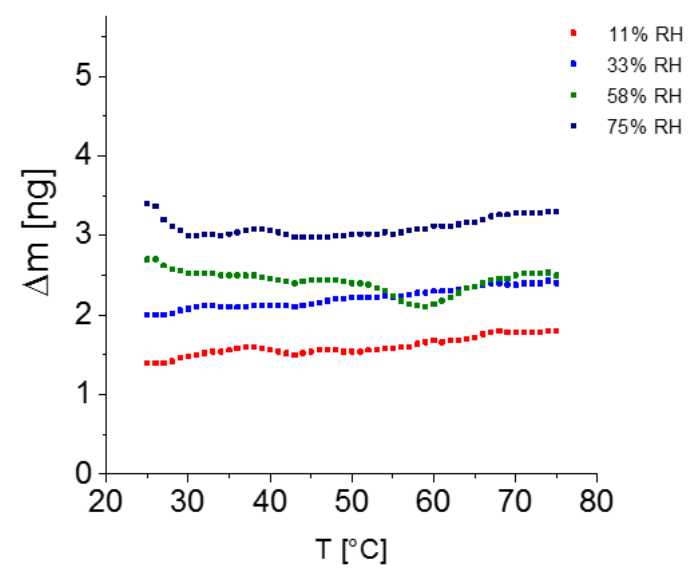

b)
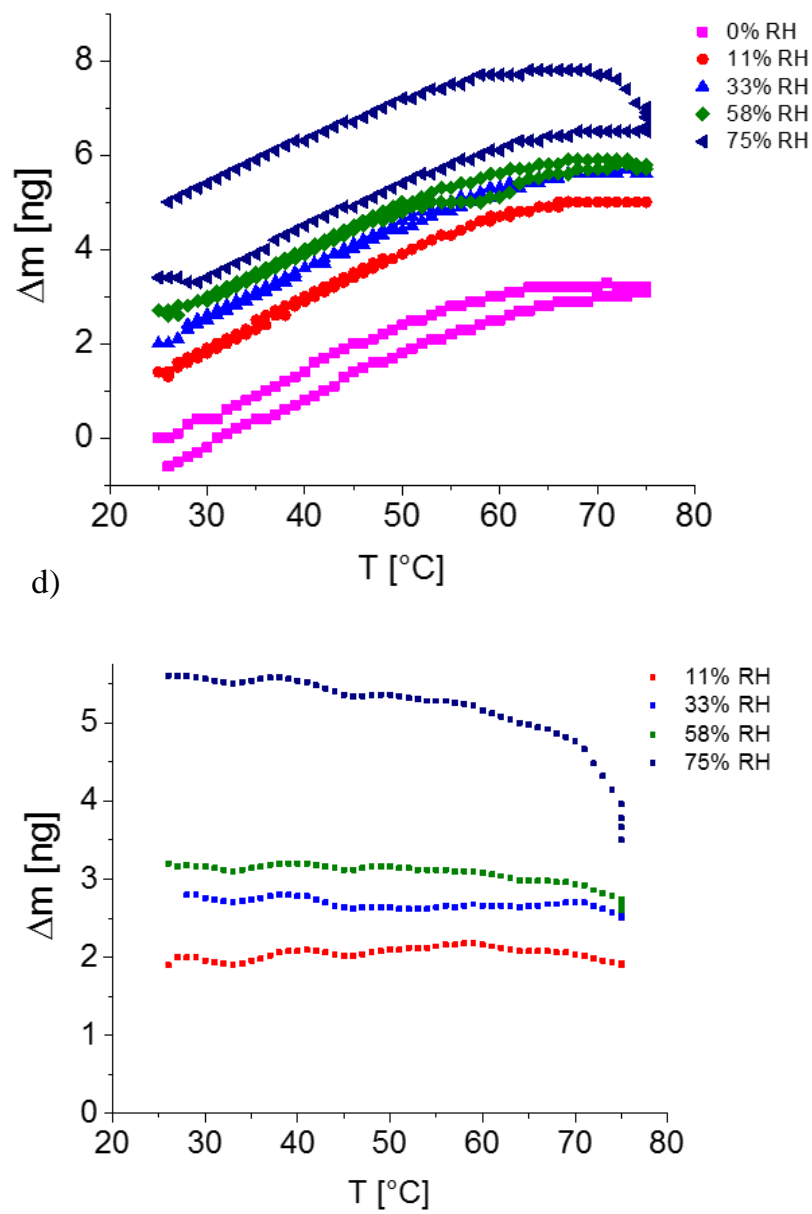

FIG. 4. (a) Mass uptake obtained from PMMA coated QCM resonance frequency shifts using the Sauerbrey model for adsorption as a function of RH. (b) Mass uptake and loss as a function of temperature during heating and cooling at constant RH with hysteresis effect due to changes in the structure of PMMA. (c) Corrected mass uptake and loss during heating and (d) cooling. The temperature dependence was removed by subtracting the reference curves acquired in the dried state.

scales larger than $10^{-12} \mathrm{~s}$ and are due to orientational fluctuations of permanent dipoles at various length scales (from bond rotations up to polymer segmental dynamics) [39].

In case of a single relaxation process, the dielectric response is well described by the Havriliak-Negami (HN) equation [40]:

$$
\varepsilon^{*}(\omega)=\varepsilon_{\infty}+\frac{\Delta \varepsilon}{\left[1+\left(\mathrm{i} \omega \tau_{\mathrm{HN}}\right)^{\alpha}\right]^{\gamma}}
$$

with $\Delta \varepsilon=\varepsilon_{\mathrm{s}}-\varepsilon_{\infty}$

Here, $\varepsilon_{\mathrm{s}}$ and $\varepsilon_{\infty}$ respectively denote the static and high frequency limiting values of the electric permittivity. The equation also contains two shape parameters $\alpha$ and $\gamma$ as well as the relaxation time $\tau_{\mathrm{HN}}$. The parameter $\Delta \varepsilon$ is the dielectric relaxation strength or intensity, which is a relevant quantity as it describes the magnitudes of the effective dipole moment. Typically, in DRS spectra, the slowest process ( $\alpha$-relaxation) is associated to the segmental motions, i.e. the dynamic glass transition $\mathrm{T}_{\mathrm{g}}$. On the other hand, the $\beta$ and $\gamma$ relaxations reflect secondary processes, which are usually attributed to side groups rotations. The
Arrhenius equation is common for describing such local dynamics relaxation time [41]:

$$
\tau=\tau_{0} \exp \left[\frac{E_{a}}{k_{b} T}\right]
$$

Here, $E_{a}$ is the activation energy of the relaxation and $\mathrm{k}_{b}$ Boltzmann's constant.

The relaxation processes were studied at different humidity conditions. The results were analyzed for each RH value in terms of water absorption and desorption as determined by the QCM data on the mass change.

\section{RESULTS}

\section{A. Kinetic QCM experiments}

Kinetics studies were carried out in the humidity and temperaturecontrolled chamber using the QCM sensor. As explained, from the acquired thickness variation, the variation of mass was calculated. 
a)

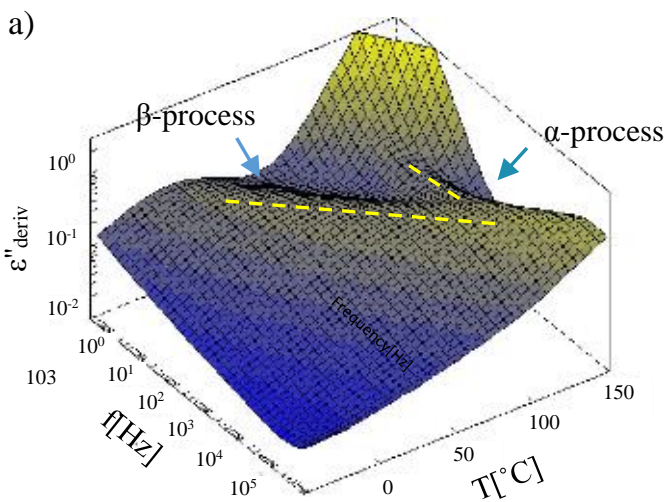

c)

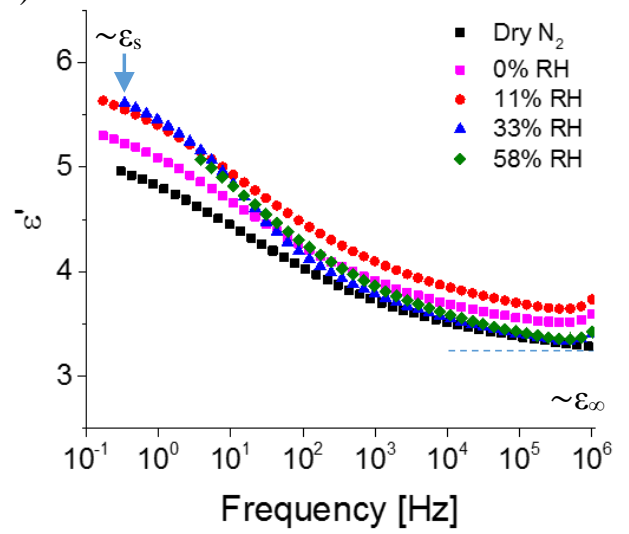

b)
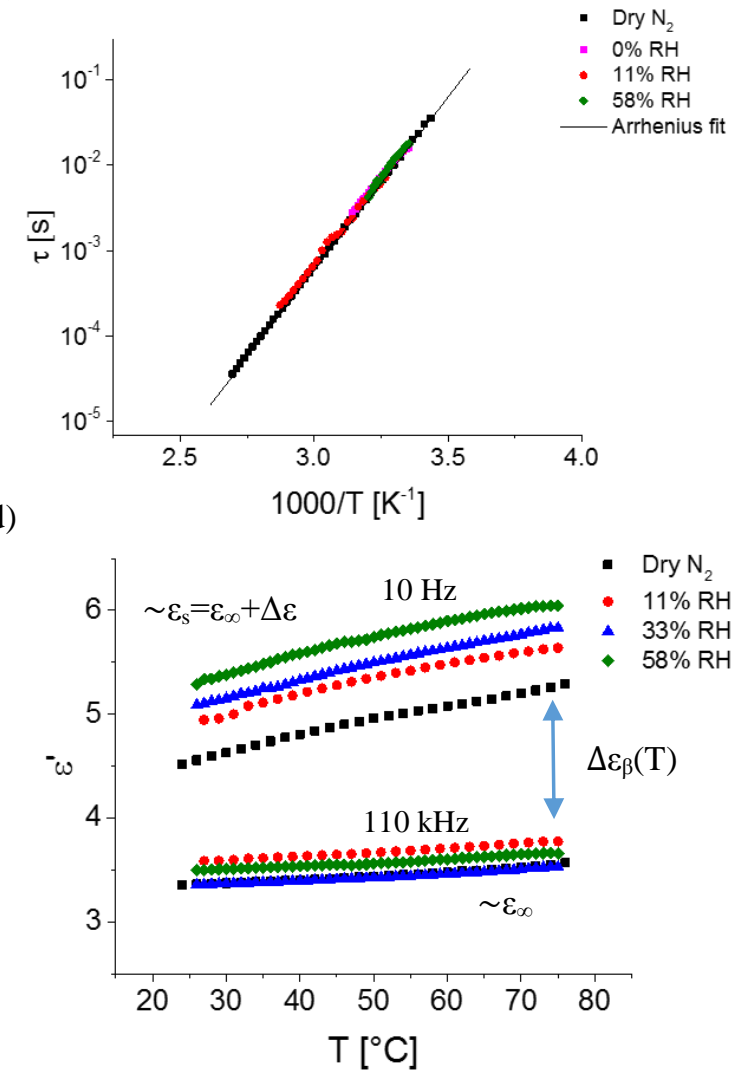

FIG. 5. (a) 3D plot of 'the conduction-free' loss $\varepsilon$ '(f) of PMMA at room condition showing the $\alpha$-relaxation process due to the motion of the main polymer chain and the $\beta$-relaxation process (b) fitted using the Arrhenius model. (c) Experimental isothermal spectra of the dielectric constant $\varepsilon^{\prime}(\mathrm{f})$ at $30^{\circ} \mathrm{C}$ and $(\mathrm{d})$ measured $\varepsilon^{\prime}(\mathrm{f})$ at $\mathrm{f}=10 \mathrm{~Hz}$ and $\mathrm{f}=110 \mathrm{kHz}$ for different humidity conditions as a function of temperature. At low frequency the secondary $\beta$-relaxation process is affected by the water content and an increase in dipole strength is observed due to a change in the mass density.

Regarding the frequency responses the Sauerbrey equation was applied. As shown in Figure $3 \mathrm{a}, \Delta \mathrm{f}$ changes significantly as a function of humidity. The first data acquired at $0 \%$ and $11 \% \mathrm{RH}$ reveal a slower desorption rate compared to the adsorption rate of the film. Between each measurement the hydration cell was opened to change the salt solution and the sample was exposed to the ambient air. This caused water uptake from the ambient air as we can see in the frequency steps for each RH condition. According to the Sauerbrey model, the frequency shift is directly proportional to the change in the adsorbed mass. Such change in the mass due to humidity adsorption was fitted using the kinetic Langmuir adsorption isotherm model as shown in Figure 3b. This model describes the rate of surface reaction for forming a monolayer on the surface [38]. The time dependence of the amount of water molecules onto the film surface $\Delta \mathrm{m}$ can be described as following:

$\Delta m_{t}=\Delta m_{0}+\Delta m_{\infty}\left(1-e^{-t / \tau}\right)$

with $\tau^{-1}=k_{a}+k_{d}$

Here, $\tau$ is the relaxation time, $m_{\infty}$ the maximum amount of water molecules adsorbed onto the surface at $\mathrm{t} \rightarrow \infty$ and $\mathrm{k}_{\mathrm{a}}$ and $\mathrm{k}_{\mathrm{d}}$ are the rate constants for the adsorption and desorption processes, respectively.

\section{B. QCM adsorption studies in quasi-equilibrium}

Figure 4a shows that the mass uptake obtained from QCM resonance frequency shifts for adsorption for different $\mathrm{RH}$ (between 0 and $75 \%$ ) values at different temperatures. Once the sample was dried and equilibrated in $\mathrm{P}_{2} \mathrm{O}_{5}$, the QCM sensor was set to zero. To ensure the system is in equilibrium, the samples were allowed to stabilize overnight at each respective $\mathrm{RH}$ condition.

The results show that the calculated mass changes as a function of the relative humidity and water uptake at higher $\mathrm{RH}$ is up to $10 \mathrm{ng}$. This curve represents an absorption isotherm showing an initial strong increase in the mass of the film, followed by a weaker linear step up to $60^{\circ} \mathrm{C}$ where the water uptake seems to be influenced by the temperature. This non-linear behavior from 0 to $10 \%$ RH might be explained by the difficult formation of polymerwater hydrogen bonds. To overcome this negative entropy effect a certain amount of favorable initial bonds needs to be formed [42]. Figure $4 \mathrm{~b}$ shows first of all that the mass upon desorption returns back to its original value. Secondly, a region of hysteresis suggests changes in the solid state structure especially at higher RH.

Even at $0 \% \mathrm{RH}$ some solvent seems to be trapped inside the polymer structure. This phenomenon is due to thermal lag in the temperature equilibration and to the kinetics of hydration. A temperature dependence is observed in Figure $4 \mathrm{~b}$ in the constant 
slope of the curves because it reflects the temperature effect on the resonance frequency of the crystal. The data have been then corrected and the $\mathrm{T}$ dependence removed by subtracting to each data set the reference data got at the dried state $\left(\mathrm{P}_{2} \mathrm{O}_{5}\right)$. As a result, we obtain the mass variation curves as function of RH shown in Figure 4c-d, which are no longer affected by heating and cooling. Regarding the measurement in $\mathrm{NaBr}(58 \% \mathrm{RH})$, the graph $4 \mathrm{c}$ shows a mass decrease around $50^{\circ} \mathrm{C}$ due to its humidity fixed points that change dramatically at higher temperature. A previous study confirms such hypothesis, in fact it reveals that despite having the same relative humidity, water content varies considerably with temperature [43]. All the relative humidity curves show the same trend and the saturation vapor pressure, thus the moisture content, is highly nonlinear as it increases rapidly with increasing temperature.

\section{Dynamics experiments}

Simultaneously, the dielectric data were acquired using IDEs. The experimental setup was validated against a conventional Novocontrol high resolution dielectric spectrometer in dry $\mathrm{N}_{2}$ atmosphere, where the sample is supposed to be water-free.

Figure 5a shows the data obtained by the conventional spectrometer, measuring in a wide temperature range from $0^{\circ} \mathrm{C}$ to $150^{\circ} \mathrm{C}$. Two processes are observed, the $\alpha$ and the $\beta$ relaxation process. Concerning the first process, it was detected, as expected, at high temperature, since the $\mathrm{T}_{\mathrm{g}}$ of s- PMMA is around $120{ }^{\circ} \mathrm{C}$. In the new setup, due to the use of saturated salt solution we could not reach such elevated temperature therefore we focus on the secondary process that occurs between $25^{\circ} \mathrm{C}$ and $75^{\circ} \mathrm{C}$. The $\beta$ relaxation process was observed at each humidity condition and follows the Arrhenius behavior below $\mathrm{T}_{\mathrm{g}}$ with an activation energy of $76 \mathrm{~kJ} / \mathrm{mol}$, which is in good agreement with the reported value $[44,45]$ (Figure $5 b)$. Such relaxation is symmetric in $\log$ frequency and is attributed to the side chain motion of PMMA e.g. the rotation of carboxyl side group is involved.

Therefore, the water uptake in weight is less than $5 \%$ of the polymer and up to $33 \%$ RH it does not affect the PMMA dynamics since the glass transition temperature is higher than the conditioning temperature of $25^{\circ} \mathrm{C}$. It also does not play a role in the activation energy of the process as shown in Figure 5c.

Important features are revealed in Figure 5d. First, at $10 \mathrm{~Hz}$ the curves reproduce the trend and the increase shown in the reference data points acquired in the dry $\mathrm{N}_{2}$ atmosphere using the conventional dielectric spectroscopy setup. Second, the dielectric constant increases in function of the adsorbed moisture content thus it is humidity dependent. Moreover, such adsorption is unaffected by temperature.

Finally, it is possible to approximate the behavior of the dielectric constant at high and low frequency to the infinite and static electric permittivity, $\varepsilon_{\infty}$ and the $\varepsilon_{s}$ respectively. The difference between both values corresponds to the relaxation strength of the $\beta$ process $\Delta \varepsilon_{\beta}$. Note that at low frequency the dielectric constant is much more influenced by the water content as its value increases. Therefore, the process appears stronger due to an increase of dipoles in the structure. As confirmed by the QCM sensor, a mass change occurs due to the water-polymer coupling.

\section{CONCLUSION}

The response of a PMMA film to changes in relative humidity was analyzed using the developed and tested compact device. The combination of simultaneous DRS and gravimetric measurements allows monitoring the film dynamics upon variation of the sample mass, which is directly related to the moisture uptake/loss.

The new equipment has a great potential thanks to its capability to simultaneously obtain several parameters from different sensors. It can bring an additional value to a variety of applications including the study of polymers and complex systems such as lipid vesicles. In addition, the setup will be improved for better controlling the humidity condition inside the chamber.

\section{ACKNOWLEDGMENTS}

This study was financed by the KU Leuven C1 project C14/15/066 "Smart Cellular Scaffolds" and by a joint project supported by the Research Foundation of Flanders (FWO) and the Ministry of Science, Technology and Productive Innovation, Argentina (MINCyT) the "Effects of Osmotic Stress on Phospholipid Membranes"; application number: VS05316N.

\section{REFERENCES}

${ }^{1}$ M. Yang, W. Su, K. Zhao 'Quantification of solvent water and hydration dynamic of thermo-sensitive microgel by dielectric spectroscopy', Polymer Phys. 55 (24), 1859-1864, 2017.

${ }^{2}$ J. L. MacCallum, A. Perez and K. A. Dill 'Determining protein structures by combining semireliable data with atomistic physical models by Bayesian inference', PNAS 112 (22), 6985-6990, 2015.

${ }^{3}$ K. A. Dill, J. L. Maccallum, 'The Protein-Folding Problem, 50 Years On', Science 338 (6110), 1042-1046, 2012.

${ }^{4}$ F. Khabaz, S. Mani, and R. Khare 'Molecular Origins of Dynamic Coupling between Water and Hydrated Polyacrylate Gels', Macromolecules 49 (19), 7551-7562, 2016.

${ }^{5}$ G. Paradossi, I. Finelli, F. Natali, M. T. F. Telling and E. Chiessi 'Polymer and water dynamics in Poly(vinyl alcohol)/Poly(methacrylate) networks. A molecular dynamics simulation and incoherent neutron scattering investigation', Polymers, 3, 1805-1832, 2011.

${ }^{6}$ F. Mallamace, P. Baglioni, C. Corsaro, S. Chen and D. Mallamace, C. Vasi, H. E. Stanley 'The influence of water on protein properties', J. Chem. Phys.141 (16), 165104, 2014.

${ }^{7}$ J. Swenson, H. Jansson, J. Hedström and R. Bergman 'Properties of hydration water and its role in protein dynamics', J. Phys.: Condens. Matter 19, 205109, 2007.

${ }^{8}$ F. Bruni and S. E. Pagnotta, 'Dielectric investigation of the temperature dependence of the dynamics of a hydrated protein', Phys. Chem. Chem. Phys. 6, 1912-1919, 2004.

${ }^{9}$ D. Laage, T. Elsaesser and J. T. Hynes, 'Water dynamics in the hydration shells of biomolecules', Chem Rev. 117(16), 10694-10725, 2017.

${ }^{10}$ E. A. Disalvo, F. Larion, F. Martini, E. Tymczyszyb, M. Frias, H. Almaleck, 'Structural and functional properties of hydration and confined water in membrane interfaces', Biochim. Biophys. Acta (BBA) Biomembranes 1778 (12), 2655-2670, 2008.

${ }^{11}$ C. Calero, H. E. Stanley and G. Franzese 'Structural Interpretation of the large slowdown of water dynamics at stacked phospholipid membranes for decreasing hydration level: all-atom molecular dynamics', Materials (Basel) 9(5), 319, 2016.

${ }^{12}$ H. Pfeiffer, H. Weichert, G. Klose, K. Heremans 'Hydration behaviour of POPC/C12-Bet mixtures investigated by sorption gravimetry, 31P NMR spectroscopy and X-ray diffraction', Chem. Phys. Lip. 165, 244-251, 2012.

${ }^{13}$ E. A. Disalvo 'Membrane Hydration: the role of water in the structure and function of biological membranes', ed. Springer, 2015. 
${ }^{14}$ M. Wübbenhorst, C. A. Murray, J. R. Dutcher 'Dielectric relaxations in ultrathin isotactic PMMA films and PS-PMMA-PS trilayer films', J. Eur. Phys. J. E, 12(Suppl 1), 109, 2003.

${ }^{15}$ S. Bistac, J. Schultz 'Study of solution-cast films of PMMA by dielectric spectroscopy: Influence of the nature of the solvent on $\alpha$ and $\beta$ relaxations', Int. J. Adhesion and Adhesives 17, 197-201, 1997.

${ }^{16}$ K. Sasaki, Y. Matsui, M. Miyara, R. Kita, N. Shinyashiki and S. Yagihara 'Glass transition and dynamics of the polymer and water in the Poly(vinylpyrrolidone)-water mixtures studied by dielectric relaxation spectroscopy', J. Phys. Chem. B 120, 6882-6889, 2016.

${ }^{17}$ T. Hirata, H. Matsuno, D. Kawaguchi, M. Inutsuka, T. Hirai, M. Tanaka and $\mathrm{K}$. Tanaka 'Dynamics of a bioinert polymer in hydrated states by dielectric relaxation spectroscopy', Phys. Chem. Chem. Phys. 19(2), 13891394, 2017.

${ }^{18}$ M. Miyara, I. Takashima, K. Sasaki, R. Kita, N. Shinyashiki and S. Yagihara 'The Dynamics of uncrystallized water in partially crystallized poly(ethylene glycol)-water mixtures studied by dielectric spectroscopy', Polymer J. 49, 511-518, 2017.

${ }^{19}$ I. Çapan, Ç. Tarimci, A. Hassan,T. Tanrisever, 'Characterisation and optical vapour sensing properties of PMMA thin films', Mat. Sc. Eng.: C, 29 (1), 140-143, 2009.

${ }^{20}$ D. Y. Sasaki, S. Singh, J. D. Cox, P. I. Pohl, 'Fluorescence detection of nitrogen dioxide with perylene/PMMA thin films', Sens. Actuators, B, Chem., 72, 51, 2001.

${ }^{21}$ M. Matsuguchi, Y. Sadaoka, and Y. Sakai, 'A capacitive-type humidity sensor using cross-linked Poly(methyl methacrylate) thin films', J. Electrochem. Soc.,138(6), 1862-1865, 1991.

${ }^{22}$ P. G. Su, Y. L. Sun, C. C. Lin, 'Humidity sensor based on PMMA simultaneously doped with two different salts', Sens. Act. B Chem. 113(2), 883-886, 2006.

${ }^{23}$ A. B. Petermann, T. Hildebrandt, U. Morgner, B. W. Roth, M. MeinhardtWollweber, 'Polymer based whispering gallery mode humidity sensor', Sensors, 18(7), 2383, 2018.

${ }^{24}$ J. Ascorbe, J. M. Corres, F. J. Arregui, I. R. Matias, 'Recent developments in fiber optics humidity sensors', Sensors, 17(4), 893, 2017.

${ }^{25} \mathrm{U}$. Ali, K. J. Karim, N. A. Buang 'A review of the properties and applications of Poly (Methyl Methacrylate) (PMMA)', Poly. Rev., 55(4), 678-705, 2015.

${ }^{26}$ A. Kasina, T. Putzeys, M. Wübbenhorst 'Dielectric and specific heat relaxations in vapor deposited glycerol', J. Chem. Phys. 143, 244504, 2015.

${ }^{27}$ M. Peeters, B. van Grinsven, T. J. Cleij, K. L. Jiménez-Monroy, P. Cornelis, E. Pérez-Ruiz et al. 'Label-free protein detection based on the heat-transfer method--A case study with the peanut allergen Ara h 1 and aptamer-based synthetic receptors.', ACS App. 1 Mater. Interfaces, 7(19), 10316-23, 2015.

${ }^{28}$ M. Zeilinger, H. Sussitz, W. Cuypers, C. Jungmann and P. Lieberzeit 'Mass-sensitive sensing of melamine in dairy products with molecularly imprinted polymers: matrix challenges', Sensors, 19(10), 2366, 2019.
${ }^{29}$ M. Khorshid, P. Losada-Pérez, G. Wackers, D. Yongabi, F.U. Renner, R. Thoelen, P. Wagner 'Real-time monitoring of interactions between Ebola fusion peptide and solid-supported phospholipid membranes: Effect of peptide concentration and layer geometry', Phys. in Med., 4, 1-7, 2017.

${ }^{30} \mathrm{P}$. Losada-Pérez, K. L. Jiménez-Monroy, B. van Grinsven, J. Leys, S. D. Janssens, M. Peeters, C. Glorieux, J. Thoen, K. Haenen, W. De Ceuninck, $P$. Wagner 'Phase transitions in lipid vesicles detected by a complementary set of methods: heat-transfer measurements, adiabatic scanning calorimetry, and dissipation-mode quartz crystal microbalance', Phys. Status Solidi A, 211, 1377-1388, 2014.

${ }^{31}$ S. Neupane, Y. De Smet, F. U. Renner, P. Losada-Pérez 'Quartz crystal microbalance with dissipation monitoring: a versatile tool to monitor phase transitions in biomimetic membranes', Front. Mater., 5, 46, 2018.

${ }^{32}$ N. V. H. Phan, H. F. Sussitz, P. A. Lieberzeit 'Polymerization parameters influencing the QCM response characteristics of BSA MIP', Biosensors, 4, 161-171, 2014.

${ }^{33} \mathrm{G}$. Z. Sauerbrey 'The use of quartz oscillators for weighing thin layers and for microweighing', Zeitschrift für Physik, 155, 206-222, 1959.

${ }^{34} \mathrm{~A}$. H. Harvey 'Encyclopedia of Physical Science and Technology', Third Edition, 16, 2001.

${ }^{35}$ S. Capponi, S. Napolitano, M. Wübbenhorst 'Supercooled liquids with enhanced orientational order', Nat. Commun. 3 (1233), 2012.

${ }^{36}$ A. Gennaro, D. Yongabi, O. Deschaume, C. Bartic, P. Wagner and M. Wübbenhorst 'Cell detection by surface imprinted polymers (SIPs) - a study of the sensor surface by optical and dielectric relaxation spectroscopy', IEEE Trans. on Diel. and Elec.Ins. 25(3), 816-821, 2018.

${ }^{37}$ F.E.M. O'Brien 'The control of humidity by saturated salt solution', J.Sci Inst. 25, 73-76, 1948.

${ }^{38} \mathrm{~T}$. Fuller 'Proton exchange membrane fuel cells 8 ', ECS Transaction 16(2), 2093-2099, 2008.

${ }^{39} \mathrm{M}$. Wübbenhorst, X. Zhang, and T. Putzeys, 'Polymer electrets and ferroelectrets as EAPs: characterization', Electromechanically active polymers: a concise reference, F. Carpi, ed. Springer, 2016.

${ }^{40}$ F. Kremer, A. Schönhals 'Broadband Dielectric Spectroscopy', ed. Springer, 2003.

${ }^{41}$ I. Langmuir, 'The constitution and fundamental properties of solids and liquids', J. Am. Chem. Soc. 38 (11), 2221-2295, 1916.

${ }^{42}$ H. M. L. Thijs, C. R. Becer, C. Guerrero-Sanchez, D. Fournier, R. Hoogenboom and U. S. Schubert' 'Water uptake of hydrophilic polymers determined by a thermal gravimetric analyzer with a controlled humidity chamber', J. Mater. Chem., 17, 4864-4871, 2007.

${ }^{43} \mathrm{O}$. Tetens, 'Über einige meteorologische Begriffe', Zeitschrift für Geophysik 6, 297, 1930.

${ }^{44}$ R. Bergman, F. Alvarez, A. Alegría, J. Colmenero, 'The merging of th dielectric $\alpha$ - and $\beta$-relaxations in poly-(methyl methacrylate)', J. Chem. Phys. 109 (17), 7546-7555, 1998.

${ }^{45}$ Y. Seki, R. Kita, N. Shinyashiki, S. Yagihara, M. Yoneyama, 'Molecular dynamics of Poly (methyl methacrylate) determined by dielectric relaxation spectroscopy', AIP Conf. Proc. 1518, 466-469, 2013. 